\title{
Desorption and Regeneration Characteristics for Previously Adsorbed Indium Ions to Phosphorylated Sawdust
}

\author{
Taik-Nam Kwon, Choong Jeon ${ }^{\dagger}$ \\ Department of Biochemical Engineering, Gangneung-Wonju National University, Gangneung 210-702, Korea
}

\begin{abstract}
The desorption characteristics of previously adsorbed indium ions on phosphorylated sawdust were tested by various chemical reagents such as $\mathrm{HCl}, \mathrm{HNO}_{3}, \mathrm{NaCl}$, ethylenediaminetetraacetic acid, and nitrilotriacetic acid. Among them, $\mathrm{HCl}$ was chosen as the best desorbing agent in terms of economics. The desorption efficiency of $\mathrm{HCl}$ for indium ions was about $97 \%$ at a concentration of $0.5 \mathrm{M}$. The desorption efficiency for indium ions was very high at about $94 \%$ even at a solid/liquid ratio of 10.0 , and the desorption process was quickly performed within $60 \mathrm{~min}$. The removal efficiency of indium ions in recycled phosphorylated sawdust could be maintained at $85 \%$ in the 4 th cycle.
\end{abstract}

Keywords: Desorption, Indium ions, Recovery, Regeneration, Sawdust

\section{Introduction}

The control of metal-loaded and used adsorbent after adsorption process is very important and gives useful information to allow for the economic design of an overall operation. In this sense, desorption and reutilization of the adsorbents in metal-recovery processes from water and wastewater are also important. In general, desorption can be performed by proton exchange using acids, chelating agents (ethylenediaminetetraacetic acid [EDTA], nitrilotriacetic acid [NTA]) or exchange with other ions (i.e., $\left.\mathrm{NaCl}, \mathrm{CaCl}_{2}\right)[1,2]$. The choice of desorbing agent depends on the kind of adsorbent used and the metals adsorbed. An effective desorbing agent is one that desorbs the metal entirely without degradation of the properties of the adsorbent. After metal desorption by means of some treatment, a regeneration step can be used to prevent adsorbent malfunction or loss of adsorption capacity. Also, the ability to regenerate the sorbent is very important in both batch and continuous processes, especially when expensive and selective sorbents are used.

Recently, concern for recycling valuable metals is increasing. Therefore, the recovery of indium ions mainly discharged from indium-tin oxide (ITO) has been widely studied. We have already shown that indium(III) ions could be selectively adsorbed by phosphorylated sawdust, which has economic merits compared to other adsorbents [3]. Unfortunately, however, there is little study on the recovery of the indium(III) ions and the regeneration of the adsorbent. In the present study, the characteristics for desorption and regeneration of indium(III) ions adsorbed onto phosphorylated sawdust was investigated with various desorbing agents.

\section{Materials and Methods}

The manufacturing methods, physical and chemical characteristics for phosphorylated sawdust are described in the references [3]. For the desorption experiment, previously adsorbed indium ions on phosphorylated sawdust were transferred to a flask containing $100 \mathrm{~mL}$ of desorbing agent such as $\mathrm{HCl}, \mathrm{HNO}_{3}$, EDTA, NTA, or NaCl. The mixture was shaken at $250 \mathrm{rpm}$ using a rotary shaking incubator (SI-600R; JEIO TECH, Seoul, Korea) at room temperature for $24 \mathrm{hr}$. Twenty-four hr was long enough to achieve an equilibrium state. After choosing the best desorbing agent, experiments to determine the optimal desorption conditions were performed. The effects on desorption efficiency of concentration, solid/liquid (S/L) ratio (the ratio of the added amount of adsorbent to the volume of desorbing agent), and desorption time were investigated. The desorption efficiency of indium(III) ion from the phosphorylated sawdust was calculated as the ratio between the amount of indium ions desorbed and the amount of indium ions adsorbed. In the regeneration experiment, the adsorbent metal that was eluted by the desorbing agent was thoroughly washed three times with deionized water to remove any traces of desorbing agent, and then mixed again

Received January 31, 2012 Accepted May 11, 2012

cc) This is an Open Access article distributed under the terms of the Creative Commons Attribution Non-Commercial License (http://creativecommons. org/licenses/by-nc/3.0/) which permits unrestricted non-commercial use, distribution, and reproduction in any medium, provided the original work is properly cited.
${ }^{\dagger}$ Corresponding Author

E-mail: metaljeon@gwnu.ac.kr

Tel: +82-33-640-2405 Fax: +82-33-641-2410 


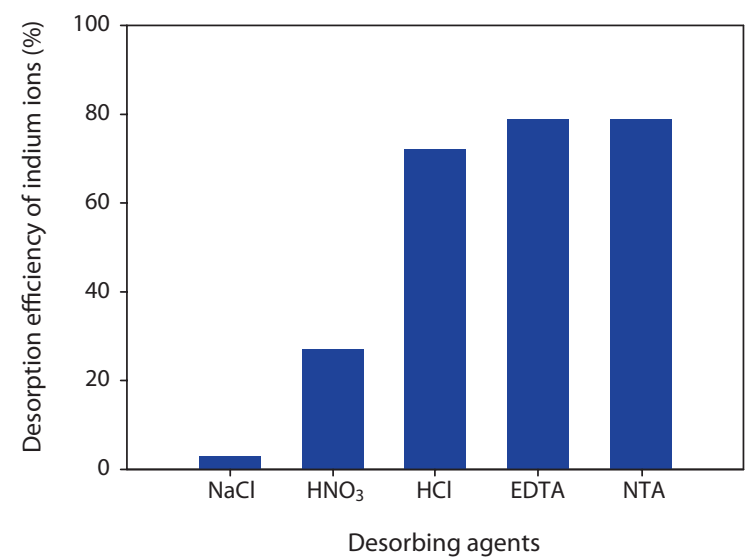

Fig. 1. Desorption efficiency of indium ion for various desorbents when initial loading of indium ions using phosphorylated sawdust was $1.075 \mathrm{mg} / \mathrm{g}$ (volume, $100 \mathrm{~mL}$; solid/liquid ratio, $1.0 \mathrm{mg} / \mathrm{mL}$; concentration of desobing agents, $0.1 \mathrm{M}$ ). EDTA: ethylenediaminetetraacetic acid, NTA: nitrilotriacetic acid.

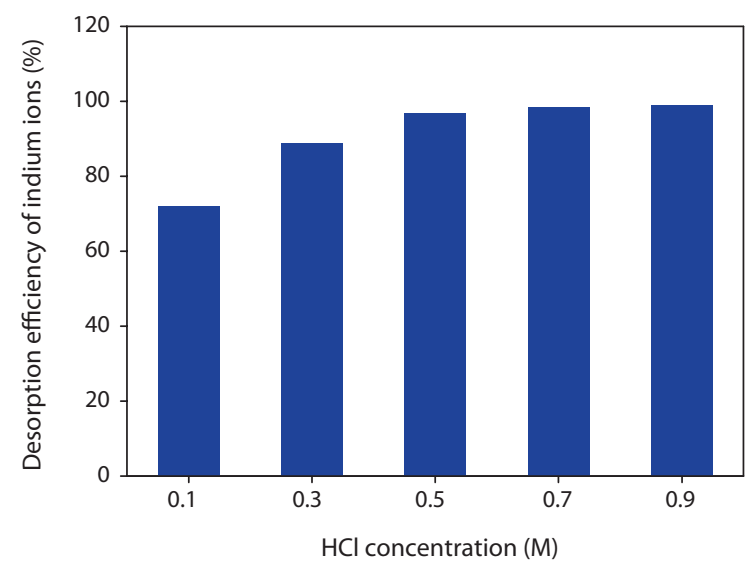

Fig. 2. Effect of $\mathrm{HCl}$ concentration on desorption efficiency of indium ions onto phosphorylated sawdust $(1.075 \mathrm{mg} / \mathrm{g}$; solid/liquid ratio, $1.0 \mathrm{mg} / \mathrm{mL}$; working volume, $100 \mathrm{~mL}$ ).

in wastewater containing indium(III) ion for the next adsorption cycle. This procedure was employed for four consecutive cycles. The removal efficiency of indium ions for each cycle was calculated using the following equation:

$$
\text { R.E }=\left(C_{i}-C_{f}\right) / C_{i},
$$

where R.E is the removal efficiency of indium ions $(\%), \mathrm{C}_{\mathrm{i}}$ is the initial concentration of indium ions $(\mathrm{mg} / \mathrm{L})$, and $\mathrm{C}_{\mathrm{f}}$ is the final concentration of indium ions $(\mathrm{mg} / \mathrm{L})$. The concentration of indium ions was analyzed by atomic absorption spectroscopy (AAnalyst 100, AAnalyst 700; PerkinElmer Inc., Waltham, MA, USA). All experiments were performed three times, and the average values are presented.

\section{Results and Discussion}

As shown in Fig. 1, in the case of EDTA and NTA, desorption

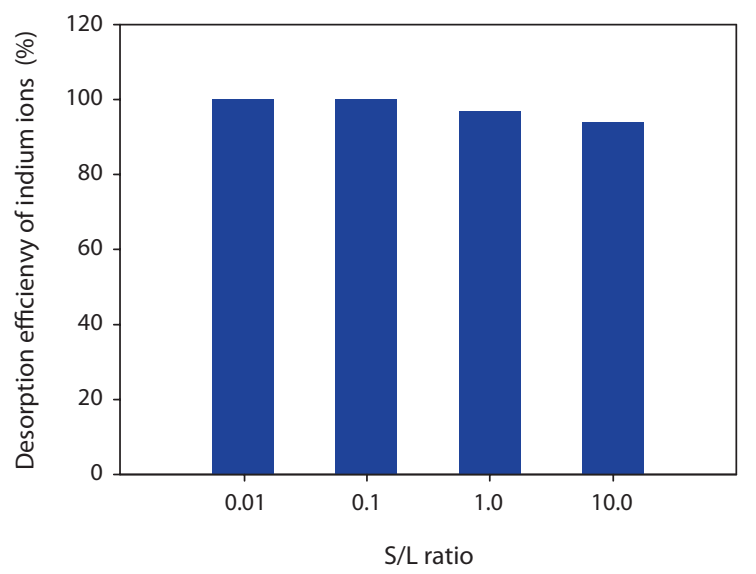

Fig. 3. Effect of solid/liquid (S/L) ratio on desorption efficiency of indium ions using $0.5 \mathrm{M} \mathrm{HCl}$ (loaded amount of indium ions onto phosphorylated sawdust, $1.075 \mathrm{mg} / \mathrm{g}$ ).

efficiencies for indium ions were about the same at around $79 \%$. On the other hand, $\mathrm{HCl}$ could desorb $72 \%$ of the indium ions. Although the desorption efficiency of $\mathrm{HCl}$ was lower than that of NTA and EDTA, the best desorbing agent for indium ions was chosen as $\mathrm{HCl}$ in terms of economic feasibility. In general, EDTA is well known as a very strong chelating agent for metal ions [4]. The investigation for the optimal concentration of $\mathrm{HCl}$ was also carried out. $\mathrm{HCl}$ concentrations of $0.1,0.3,0.5,0.7$, and $0.9 \mathrm{M}$ were tested for elution of adsorbed indium ions. The initial indium loading for phosphorylated sawdust was $1.075 \mathrm{mg} / \mathrm{g}$ dry mass [3]. Fig. 2 shows that the desorption efficiency for indium ions increased as the concentration of $\mathrm{HCl}$ increased. However, changes of desorption efficiency did not occur at $\mathrm{HCl}$ concentrations over $0.5 \mathrm{M}$. Therefore, with $97 \%$ desorption efficiency, the $0.5 \mathrm{M}$ was chosen as the optimal concentration for $\mathrm{HCl}$ in terms of cost and efficiency. As mentioned, the S/L ratio is a very important operation parameter in batch processes [5]. Various $\mathrm{S} / \mathrm{L}$ ratios including $0.01,0.1,1.0$, and 10.0 were tested. Generally, it is well known that as the S/L ratio decreases, desorption efficiency increases [6]. But in terms of the cost, there is an ideal value. Fig. 3 shows that as the S/L ratio increased, desorption efficiency decreased. At very low S/L ratio, the desorption efficiency approached $100 \%$ because much desorbing agent was used for comparatively less phosphorylated sawdust. The desorption efficiency for indium ions was very high at about $94 \%$, even at an S/L ratio of 10.0. However, to apply a desorption operation to real processes, the concentration factor (data not shown), which is defined as the ratio of desorbed metal concentration in a desorbing agent solution to the initial metal concentration in aqueous solution, must be also considered as another important parameter [7]. The concentration factor generally increased with the S/L ratio. Fig. 4 shows that the desorption efficiency for indium ions increased with time. However, most of the desorption process was completed within $60 \mathrm{~min}$. It means that desorption process was performed very quickly. Generally, it has been reported that most of the desorption processes finish within 180 min [5]. To develop a batch treatment process, it is essential to describe regeneration aspects of the process in order to improve its cost-effectiveness by recycling the adsorbent for reuse in multiple cycles. In order to investigate regeneration ability for phos- 


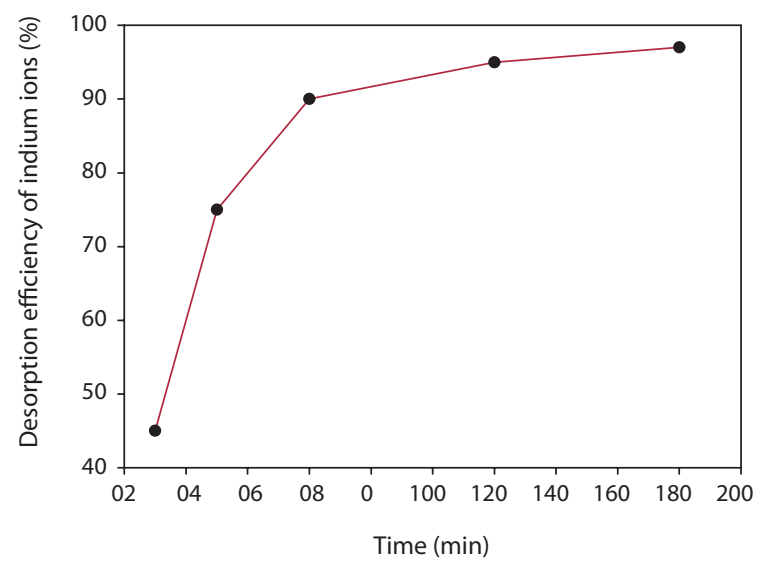

Fig. 4. Change of desorption efficiency of indium ions with time using $0.5 \mathrm{M}$ of $\mathrm{HCl}$ (solid/liquid ratio, 1.0; working volume, $100 \mathrm{~mL}$ ).

Table 1. Regeneration on indium ions for phosphorylated sawdust

\begin{tabular}{ccc}
\hline Cycle no. & $\begin{array}{c}\text { Removal efficiency of } \\
\text { indium ions (\%) }\end{array}$ & $\begin{array}{c}\text { Reduced removal } \\
\text { efficiency (\%) }\end{array}$ \\
\hline 1 & 93 & - \\
2 & 91 & 2 \\
3 & 87 & 4 \\
4 & 85 & 2 \\
\hline
\end{tabular}

$0.5 \mathrm{M}$ of $\mathrm{HCl}$ was used as a desorbing agent and $\mathrm{S} / \mathrm{L}$ ratio was set as 1.0 .

phorylated sawdust, sequential adsorption-desorption cycles were repeated 4 times using the same adsorbent. As shown in Table 1, removal efficiency of indium ions for recycled phosphorylated sawdust can be maintained at $85 \%$ even in the 4 th cycle, although there is a slight decrease in removal efficiency due to incomplete desorption for indium ions. Gupta and Babu [8] showed that regenerated sawdust exhibits higher removal efficiency at about $85 \%$ for $\mathrm{Cr}(\mathrm{VI})$. Consequently, it could be concluded that phosphorylated sawdust can be sufficiently used in real processes such as industrial wastewater discharged from ITO thin film processes.

\section{Conclusions}

Previously adsorbed indium ion on phosphorylated sawdust was effectively desorbed by HCl. Desorption efficiency for indium ion was about $97 \%$ when a concentration of $0.5 \mathrm{M}$ was used. Also, the desorption efficiency for indium ions was very high at about $94 \%$, even at an S/L ratio of 10.0, and most of desorption process was completed within $60 \mathrm{~min}$. In addition, the desorption efficiency of recycled phosphorylated sawdust for indium ions can be kept at $85 \%$ through 4 cycles.

\section{Acknowledgments}

The authors gratefully acknowledge the Korea Institute of Energy Technology Evaluation and Planning for financial support in this study.

\section{References}

1. Beolchini F, Pagnanelli F, Toro L, Veglio, F. Biosorption of copper by Sphaerotilus natans immobilized in polysulfone matrix: equilibrium and kinetic analysis, Hydrometallurgy 2003;70:101-112.

2. Sekhar KC, Kamala CT, Chary NS, Sastry AR, Rao TN, Vairamani M. Removal of lead from aqueous solutions using an immobilized biomaterial derived from a plant biomass. $J$. Hazard. Mater. 2004;108:111-117.

3. Choong J, Kwon TN. Selective adsorption for indium(III) from industrial wastewater using chemically modified sawdust. J. Ind. Eng. Chem. 2012; In press.

4. Dawson RM, Elliott DC, Elliott WH, Jones KM. Data for biochemical research. 3rd ed. Oxford: Clarendon Press; 1986.

5. Volesky B. Biosorption of heavy metals. Boca Raton: CRC Press; 1990.

6. Mustafa G, Singh B, Kookana RS. Cadmium adsorption and desorption behavior on goethite at low equilibrium concentrations: effects of $\mathrm{pH}$ and index cations. Chemosphere 2004;57:1325-1333.

7. Jeon C, Park KH. Adsorption and desorption characteristics of mercury(III) ions using aminated chitosan bread. Water Res. 2005;39:3938-3944.

8. Gupta S, Babu BV. Removal of toxic metal Cr(VI) from aqueous solutions using sawdust as adsorbent: equilibrium, kinetics and regeneration studies. Chem. Eng. J. 2009;150:352365. 